overestimation of the effect of a shorter inter-pregnancy interval. However, if male gender was indeed on the causal pathway between inter-pregnancy interval and schizophrenia, this would not, of itself, lead to a biased estimate of association between inter-pregnancy interval and schizophrenia. Furthermore, if male gender was indeed on the causal pathway, then adjusting for gender should lead to an attenuation of the association between inter-pregnancy interval and schizophrenia; however, adjusting for gender made no difference to our results, ${ }^{1}$ indicating that gender is unlikely to be an adequate explanation as a mechanism for the association with shorter inter-pregnancy interval.

1 Gunawardana L, Davey Smith G, Zammit S, Whitley E, Gunnell D, Lewis S, et al. Pre-conception inter-pregnancy interval and risk of schizophrenia. $\mathrm{Br} J$ Psychiatry 2011; 199: 338-9.

2 Chabrol H, Chauchard E, Girabet J. Cannabis use and suicidal behaviours in high-school students. Addict Behav 2008; 33: 152-5.

3 Cheslack-Postava K, Liu K, Bearman PS. Closely spaced pregnancies are associated with increased odds of autism in California sibling births. Pediatrics 2011; 127: 246-53.

4 Smits L, Pedersen C, Mortensen P, van Os J. Association between short birth intervals and schizophrenia in the offspring. Schizophr Res 2004; 70: 49-56.

5 Smits LJ, Essed GG. Short interpregnancy intervals and unfavourable pregnancy outcome: role of folate depletion. Lancet 2001; 358: 2074-7.

Stanley Zammit, Clinical Senior Lecturer in Psychiatric Epidemiology, MRC Centre for Neuropsychiatric Genetics and Genomics, Cardiff University, and School of Socia and Community Medicine, University of Bristol, UK. Email: ZammitS@cardiff.ac.uk: Lihini Gunawardana, MRC Centre for Neuropsychiatric Genetics and Genomics, Cardiff University, UK; George Davey Smith, Elise Whitley, David Gunnell Sardiff University, UK; George Davey Smith, Elise Whitley, David Gunnell, Finn Rasmussen, Department of Public Health Sciences, Karolinska Institute, Stockholm, Sweden

doi: 10.1192/bjp.200.2.160a

\section{Care clusters and mental health Payment by Results}

In their piece on mental health Payment by Results, ${ }^{1}$ Macdonald \& Elphick note 'a lack of reassurance that costs per case within a cluster will be similar enough to support a viable tariff calculation' This may underestimate the difficulties with the proposed new payment mechanism, which may have effects wider than disruption of nascent routine outcome measurement systems.

The UK has come relatively late to the process of payment reform for mental health services, but despite this it has followed an approach unlike that in other countries. The fundamental principle behind the care cluster approach seems to be the presumption that individuals with similar needs for care, as notionally defined by clusters of scores on the Health of the Nation Outcome Scales (HoNOS), will receive similar care and therefore incur similar costs. Importantly, costs themselves did not enter into the original process of defining care clusters. ${ }^{2}$

The approach pioneered by Fetter at Yale ${ }^{3}$ in developing the original Medicare prospective payment system in the 1980s was to combine consultation with clinicians and statistical analysis of clinical, administrative and cost data using variance reduction so that case-mix groupings are both expected to produce similar 'clinical responses' and also do in fact demonstrate acceptable homogeneity of costs. This approach was also followed by Australia and New Zealand, ${ }^{4,5}$ when they attempted to develop payment systems based on HoNOS. Achieving homogeneous costs within groups is crucial because it minimises the random risk to providers (the risk that appropriately incurred costs and therefore revenue differ randomly from those reimbursed). A typical cut-off for acceptable cost homogeneity is for each case-mix group to have a coefficient of variation of one or less (mean divided by standard deviation). It is also essential to make sure that factors relevant in resource use which may be more or less prevalent among different providers are also represented; otherwise there may be an element of systematic risk, with certain providers being systematically underpaid and others systematically overpaid. Even when this more standard approach is followed, it may not be successful, especially in mental health, where cost variation is high. Infamously, the original Medicare prospective payment system was never implemented in specialist mental health units in the face of evidence that resource homogeneity was too low and that the system would systematically disadvantage those units, and has now been abandoned in favour of an across the board per diem payment system for psychiatric in-patients. ${ }^{6}$ Neither the Australian nor New Zealand systems were ever implemented.

In the light of the foregoing comments, it is perhaps not surprising that the Department of Health's own pilot studies from 2006 demonstrate both that resource homogeneity of care clusters is unacceptably low, with only 1 of 13 clusters having a coefficient of variation of less than one, and also that far better homogeneity could have been achieved, especially for in-patients, had the standard variance-reduction approach been followed. ${ }^{7}$ At present, it seems to me that the lowest risk approach to reforming payment for mental health services is to adopt a basic system of activitybased funding, and use the data collected in this way, along with clinical and administrative data, as part of a slow and careful process of reform. Certainly, payment for mental health services in the UK is ripe for reform, as variations in resource use between providers are far wider than could be accounted for by any difference in case-mix. ${ }^{8}$ But this may not be the best way of approaching it.

1 Macdonald AJD, Elphick M. Combining routine outcomes measurement and 'Payment by Results': will it work and is it worth it? Br J Psychiatry 2011; 199: 178-9.

2 Self R, Rigby A, Leggett C, Paxton R. Clinical Decision Support Tool: a rational needs-based approach to making clinical decisions. J Ment Health 2008; 17: 33-48.

3 Fetter RB. Health Care Financing Grants and Contracts Report: The New ICD-9-CM Diagnosis Related Groups Classification Scheme. Health Care Financing Administration, 1983. Available from: http://babel.hathitrust.org/ cgi/pt?id=mdp .39015009571160 .

4 Buckingham B, Burgess $\mathrm{P}$, Solomon $\mathrm{S}$, Pirkis JE, Eagar K. Developing a Casemix Classification for Mental Health Services. Volume 1: Main Report. Commonwealth Department of Health and Family Services, 1998.

5 Eagar K, Gaines P, Burgess P, Green J, Bower A, Buckingham B, et al. Developing a New Zealand casemix classification for mental health services. World Psychiatry 2004; 3: 172-7.

6 Lave JR. Developing a Medicare prospective payment system for inpatient psychiatric care. Health Aff 2003; 22: 97-109.

7 Health and Social Care Information Centre Casemix Service. Mental Health Casemix Classification Development: End Stage Report. Health and Social Care Information Centre, 2006.

8 Audit Commission. Maximising Resources in Adult Mental Health. Audit Commission, 2010.

Alex D. Tulloch, BRC Preparatory Clinician Scientist Fellow, Institute of Psychiatry, King's College London, UK. Email: alex.tulloch@kcl.ac.uk

doi: 10.1192/bjp.200.2.161

The key to doing routine mental health outcomes well ${ }^{1}$ is to make them relevant, meaningful and available to practitioners, service users and managers. The Health of the Nation Outcome Scales (HoNOS) is now a front-runner for a general outcome measure since it is required for Payment by Results, a new contracting system for mental healthcare in the UK. Only one HoNOS rating is currently required in order to allocate patients to Payment by Results care clusters, so managers have little incentive to take the extra step and mandate more than one HoNOS rating to assess the effectiveness of interventions. The simplest way to introduce outcome measurement with HoNOS would be to mandate at least two ratings, one at the outset of an intervention and one 
at the close. A benchmark for this approach has been set by the Priory Group for HoNOS outcomes of in-patient stays (www.priorygroup.com/Personal-Site/About-Priory/AboutUs/Healthcare-Outcomes/General-Psychiatry.aspx). Psychological therapists are ahead of the curve, since many already use a commercial outcomes tool (e.g. Clinical Outcomes in Routine Evaluation, CORE; www.coreims.co.uk) in their work to monitor treatment progress, to support reflection and to aid supervision. They also involve patients, who make their own ratings on a standard questionnaire. They have made outcomes relevant and meaningful. Could their experience help develop HoNOS as an outcome tool? The HoNOS could be put to work supporting practitioners. For example, HoNOS could inform referral and assessment systems, by helping select between primary and secondary care services. If no individual HoNOS item rating is greater than 2 (mild), then secondary services may not be indicated. Individual scale scores could also indicate priorities for interventions: a high score on 'hallucinations and delusions' and a low score on 'living conditions' could suggest a focus on treatment over accommodation (and vice versa). The HoNOS total and individual scale scores would also indicate progress and could be used in supervision. The HoNOS scores that fall below predetermined thresholds may indicate readiness for discharge. These could even be agreed as goals with patients. Co-producing HoNOS with service users and carers could balance the perspective of HoNOS as a clinician-rated measure. Getting all practitioners on board will need vision and effort. Gilbody et $a l^{2}$ found that psychiatrists were not very interested in recording standardised outcomes. Feedback is crucial to engagement. Trusts should invest time to design their information systems so that they report person-centred outcomes directly to practitioners and teams in a meaningful format. Simply reporting outcome returns centrally would miss a huge opportunity to engage clinicians with outcomes, but still burden them with data collection. Outcomes information will create new challenges, for example the apparent ability to compare the effectiveness of teams and individual practitioners. For some, this could be intensely motivating or intimidating. The introduction of standard outcome measures should be done thoughtfully with ongoing input from service users, practitioners, managers and academics; or as Macdonald \& Elphick put it: well.

\section{Declaration of interest}

Outcomes data affect my appraisal. I have a clinical information lead role in my trust.

1 Macdonald AJD, Elphick M. Combining routine outcomes measurement and 'Payment by Results': will it work and is it worth it? Br J Psychiatry 2011; 199: $178-9$

2 Gilbody SM, House AO, Sheldon TA. Psychiatrists in the UK do not use outcomes measures. National survey. Br J Psychiatry 2002; 180: 101-3.

David Yeomans, psychiatrist, Leeds Partnerships NHS Foundation Trust, UK. Email: david.yeomans@nhs.net

doi: 10.1192/bjp.200.2.161a

MacDonald \& Elphick ${ }^{1}$ have lucidly described the proposed introduction of Payment by Results into mental health. They mention, however, that 'concerns include the validity and reliability of the MHCT' (mental health clustering tool), and there is also the major issue of how cost can be firmly linked to the quality of services delivered.

The Department of Health approach to reliability has been to rely on local initiatives and to commission the development of an algorithm based on the MHCT to ensure that clusters are reliably allocated. Exactly why the Department of Health believes that this is possible by either route is not clear. Local initiatives are the route to mayhem and none of the attempts at devising algorithms so far have been successful. The instrument on which the MHCT is based, Health of the Nation Outcome Scales (HoNOS), was not devised for this purpose. Additional items have been added but this was for clinical reasons. The HoNOS was devised as a clinical outcome measure, not for needs assessment and certainly not as a classification tool. Internationally recognised tools (e.g. Schedules for Clinical Assessment in Neuropsychiatry, Structured Clinical Interview for DSM Disorders) have been devised to classify conditions but these use a range of information (e.g. symptoms, beliefs and timescales), with specified criteria that have been and continue to be subject to international expert scrutiny.

A unit of costing must be directly related to quality and outcome measures or the UK will have the same problems as the USA have encountered in its payment systems. It is difficult to understand how clusters can be such units of cost unless there is a very substantial body of research investigating evidence for efficacy of interventions (e.g. cognitive therapy and medication) for individual clusters, and for the development and reliability testing of outcome measures - which would take years. Attempting to match cluster to pathway/intervention has to be done by using diagnosis as an intermediate step because that is where the evidence currently exists. The problem then is that each cluster relates to a number of guidelines and monitoring quality becomes complicated - as trusts, and previously the Department of Health, are finding in attempting to devise cluster pathways. General practice commissioners won't have the time, resource or inclination to undertake such complex monitoring - so cost will rule.

Broad diagnoses, as used by the National Institute for Health and Clinical Excellence, have proved satisfactory for clinical purposes and have readily available, reliable and applicable outcome measures $^{2}$ and, although diagnosis alone is not sufficient for costing, in combination with clinical pathways ${ }^{3}$ they can be costed and used for tariffs with a much better chance of reliability and homogeneity. The very limited data that have been produced so far are promising (available on request) but there needs to be more extensive examination of data. The Department of Health needs to take a lead because trusts are not going to re-analyse their data using diagnosis and allocation to pathways unless the Department of Health asks them to do so.

As MacDonald \& Elphick ${ }^{1}$ describe, outcome measurement is needed in any system and clustering has been very effective at promoting use of HoNOS. However, combining diagnosis and pathways could provide a simpler, practical approach to gathering data for costing and tracking change than can making use of clusters. It would also lead to an improved quality of care by linking cost directly to the use of evidence-based guidelines and care pathways by empowered patients, carers, providers and commissioners.

1 MacDonald AJD, Elphick M. Combining routine outcomes measurement and Payment by Results: will it work and is it worth it? Br J Psychiatry 2011; 199: 178-9.

2 NHS South Central. Emotional Wellbeing. NHS South Central, 2011 (http:// www.sha.nhsdigital.org.uk/index.php?option=com_content\&view=article\& $\mathrm{id}=52$ \&ltemid $=33$ ).

3 NHS South Central. What services can I expect? NHS South Central, 2011 (http://www.sha.nhsdigital.org.uk/index.php?option=com_content\&view= article\&id=203\&/temid=31). David G. Kingdon, University of Southampton, UK. Email: dgk@soton.ac.uk; Bohdan
Solomka, Norfolk and Waveney Mental Health NHS Foundation Trust; Hamish Solomka, Norfolk and Waveney Mental Health NHS Foundation Trust; Hamish
McAllister-Williams, Douglas Turkington, University of Newcastle upon Tyne; Alain Gregoire, Hesham Elnazer, Mahesh Thagadur, Lars Hansen, Shanaya Rathod, Stefan Gleeson, Southern Health NHS Foundation Trust; Mo Zoha, Central \& North West London NHS Foundation Trust; Pratima Singh, South London \& Maudsley NHS Foundation Trust; Farooq Naeem, Isle of Wight NHS Primary Care Trust

doi: 10.1192/bjp.200.2.162 